\title{
Generalizing the soldering procedure
}

\author{
D. Dalmazi, ${ }^{1}$ A. de Souza Dutra, ${ }^{1}$ and E. M. C. Abreu $^{2}$ \\ ${ }^{1}$ UNESP-Campus de Guaratinguetá-DFQ, Av. Dr. Ariberto Pereira da Cunha, 333 CEP 12516-410 - Guaratinguetá - SP - Brazil. \\ ${ }^{2}$ Universidade Federal Rural do Rio de Janeiro, Departamento de Fáísica BR 465 km 07 Centro Seropédica CEP 23890-000 RJ Brazil
} (Received 28 March 2006; published 19 July 2006)

\begin{abstract}
We start this work by revisiting the problem of the soldering of two chiral Schwinger models of opposite chiralities. We verify that, different from what one can conclude from the current literature, the usual sum of these models is, in fact, gauge invariant and corresponds to a composite model, where the component models are the vector and axial Schwinger models. As a consequence, we reinterpret this formalism as a kind of degree of freedom reduction mechanism. This result has led us to discover a second soldering possibility giving rise to the axial Schwinger model. This new result is seemingly rather general. We explore it here in the soldering of two Maxwell-Chern-Simons theories with different masses.
\end{abstract}

DOI: 10.1103/PhysRevD.74.025015

PACS numbers: 11.15.- q, 11.10.Ef

\section{INTRODUCTION}

The study of chiral bosonic fields has arisen and proliferate mainly due to its importance in the quantization of strings [1] and other theoretical models [2]. This research in chiral bosonization has begun many years back with the seminal paper of W. Siegel [3]. Floreanini-Jackiw have offered later some different solutions to the problem of a single self-dual field [4] proposing a nonanomalous model. The study of chiral bosons also plays an important role in the studies of the quantum Hall effect [5]. The introduction of a soliton field as a charge-creating field obeying one additional equation of motion leads to a bosonization rule [6]. The author of [7] has shown that the method of coadjoint orbit [8], when applied to a representation of a group associated with a single affine Kac-Moody algebra, generates an action for the chiral WZW model [9], which is a non-Abelian generalization of the Floreanini and Jackiw (FJ) model.

The concept of soldering [7,10,11] has proved extremely useful in different contexts. The soldering formalism essentially combines two distinct Lagrangians manifesting dual aspects of some symmetry to yield a new Lagrangian which is destituted of, or rather hides, that symmetry. The quantum interference effects, whether constructive or destructive, among the dual aspects of symmetry, are thereby captured through this mechanism [11]. The formalism introduced by M. Stone [7] could actually be interpreted as a new method of dynamical mass generation [11]. This technique parallels a similar phenomenon in two dimensional field theory known as Schwinger mechanism [12] that results from the interference between right and left massless self-dual modes of chiral Schwinger model [13] of opposite chiralities [11]. The result of the chiral interference shows the presence of a massive vectorial mode, for the special case where the Jackiw-Rajaraman regularization parameter is $a=1$ [13], which is the value where the chiral theories have only one massless excitation in the spectrum. This clearly shows that the massive vector mode results from the interference between two massless modes.
It was shown lately [14], that in the soldering process of two Siegel's [3] modes (lefton and righton) coupled to a gauge field [15], this gauge field has decoupled from the physical field. The final action describes a nonmover field (a noton) at the classical level. The noton acquires dynamics upon quantization. This field was introduced by Hull [16] to cancel out the Siegel anomaly. It carries a representation of the full diffeomorphism group, while its chiral components carry the representation of the chiral diffeomorphism.

In the $3 D$ case, the soldering mechanism was used to show the result of fusing together two topologically massive modes generated by the bosonization of two massive Thirring models with opposite mass signatures in the long wave-length limit. The bosonized modes, which are described by self and anti-self-dual Chern-Simons models $[17,18]$, were then soldered into the two massive modes of the $3 D$ Proca model [19]. In the $4 D$ case, the soldering mechanism produced an explicitly dual and covariant action as the result of the interference between two SchwarzSen [20] actions displaying opposite aspects of the electromagnetic duality [19].

In this work we revisit the problem of the soldering of two chiral Schwinger models of opposite chiralities. Verifying that the usual sum of these models is, in fact, gauge invariant and corresponds to a composite model, where the component models are the vector and axial Schwinger models [21]. So, in this particular case, we show that it is not really necessary to use the soldering mechanism to accomplish the gauge symmetry as supposed. As a consequence, we reinterpret it as a kind of degree of freedom reduction mechanism. This idea is then used in order to define other possible ways of performing this soldering/fusion procedure.

\section{A BRIEF DESCRIPTION OF THE SOLDERING FORMALISM}

The soldering formalism gives an useful bosonization scheme for Weyl fermions, since a level one representation 
of $\mathrm{LU}(\mathrm{N})$ has an interpretation as the Hilbert space for a free chiral fermion [22]. However, only Weyl fermions can be analyzed in this way, since a $2 D$ conformally invariant QFT has separated right and left current algebras. In other words, it is trivial to make a (free) Dirac fermion from two (free) Weyl fermions with opposite chiralities. The action is just the sum of two Weyl fermion actions. It seems, however, nontrivial to get the action of the WZW model from two chiral boson actions of opposite "chiralities", because it is not the direct sum of two chiral bosons.

To solve this problem, Stone [7] introduced the idea of soldering the two chiral scalars by introducing a nondynamical gauge field to remove the degree of freedom that obstructs the vector gauge invariance [23]. This is connected, as we said above, to the necessity that one must have more than the direct sum of two fermions representations of the Kac-Moody algebra to describe a Dirac fermion. In another way we can say that the equality for the weights in the two representations is physically connected with the necessity to abandon one of the two separate chiral symmetries, and accept that a nonchiral gauge symmetry should be kept. This is the main motivation for the introduction of the soldering field which makes possible the fusion of dualities in all space-time dimensions. This restriction will force the two independent chiral representations to belong to the same multiplet, effectively soldering them together.

The basic idea of the soldering procedure is to raise a global Noether symmetry of the self and anti-self-dual constituents into a local one. The effective theory, consists of the dual components and an interference term [23].

An iterative Noether procedure [23] is usually adopted in order to promote global symmetries. Therefore, one supposes that the symmetries in question are being described by the local actions, invariant under a global transformation. Then, trying to raise the symmetry to a local one, notice that now under local transformations these actions will not remain invariant, and Noether counterterms become necessary to reestablish the invariance, along with appropriate auxiliary fields, the so-called soldering fields which by construction should be nondynamical ones.

For each the self and anti-self-dual system we have in mind that this iterative gauging procedure is constructed not to produce invariant actions for any finite number of steps. However, if after $\mathrm{N}$ repetitions, the noninvariant piece ends up being only dependent on the gauging parameters and Noether currents, then there will exist the possibility of mutual cancelation if both self and antiself gauged systems are combined with each other.

Finally, the auxiliary fields should be eliminated, for instance, through its equations of motion, from the resulting effective action, in favor of the physically relevant degrees of freedom. It is important to notice that after the elimination of the soldering fields, the resulting effective action will not depend on either self or anti-self-dual fields but only on some collective field, defined in terms of the original ones in a invariant way.

\section{THE CHIRAL SCHWINGER MODEL}

Let us begin by introducing the notation used here for the light cone variables:

$$
\begin{gathered}
x_{ \pm}=\frac{1}{\sqrt{2}}\left(x_{0} \pm x_{1}\right), \quad \partial_{ \pm}=\frac{1}{\sqrt{2}}\left(\partial_{0} \pm \partial_{1}\right), \\
A_{ \pm}=\frac{1}{\sqrt{2}}\left(A_{0} \pm A_{1}\right),
\end{gathered}
$$

and now we can work out our model.

We can write down the interaction terms of the chiral Schwinger model for both chiralities in its bosonized form as:

$$
W_{+}=\int d^{2} x\left(\partial_{+} \phi \partial_{-} \phi+e A_{+} \partial_{-} \phi+\frac{a e^{2}}{4} A_{+} A_{-}\right)
$$

and

$$
W_{-}=\int d^{2} x\left(\partial_{+} \rho \partial_{-} \rho+e A_{-} \partial_{+} \rho+\frac{b e^{2}}{4} A_{+} A_{-}\right),
$$

where $a$ and $b$ are the Jackiw-Rajaraman coefficients for each chirality respectively [13]. Notice that $W_{+}$and $W_{-}$ are invariant under the following semilocal gauge transformations respectively

$$
\begin{aligned}
& \delta A_{+}=0 ; \quad \delta A_{-}=\partial_{-} \epsilon_{R} ; \quad \delta \phi=-a e \epsilon_{R} / 4 \\
& \delta A_{+}=\partial_{+} \epsilon_{L} ; \quad \delta A_{-}=0 ; \quad \delta \phi=-b e \epsilon_{L} / 4
\end{aligned}
$$

where $\epsilon_{R}=\epsilon_{R}\left(x_{-}\right)$and $\epsilon_{L}=\epsilon_{L}\left(x_{+}\right)$. Performing a direct sum of the actions we have:

$$
\begin{aligned}
W_{\mathrm{TOTAL}}= & W_{+} \oplus W_{-} \\
= & \int d^{2} x\left(\partial_{+} \phi \partial_{-} \phi+\partial_{+} \rho \partial_{-} \rho+e A_{+} \partial_{-} \phi\right. \\
& \left.+e A_{-} \partial_{+} \rho+\frac{(a+b) e^{2}}{4} A_{+} A_{-}\right),
\end{aligned}
$$

Notice that if $a+b=2, W_{\text {TOTAL }}$ is invariant under $\delta \rho=$ $\epsilon=\delta \phi ; \delta A_{ \pm}=-2 \partial_{ \pm} \epsilon$ where $\epsilon=\epsilon\left(x_{\mu}\right)$ is an arbitrary function. In order to make this local gauge invariance explicit, let us do the following rotation

$$
\begin{aligned}
\sqrt{2} \rho & =\sigma+\varphi \\
\sqrt{2} \phi & =\sigma-\varphi .
\end{aligned}
$$

Substituting in (6) and writing the result in a explicit covariant way we have a vector plus an axial Schwinger model:

$$
\mathcal{L}=\mathcal{L}_{\mathrm{VSM}}+\mathcal{L}_{\mathrm{ASM}}+\frac{1}{4}(a+b-2) e^{2} A_{\mu}^{2}
$$


with

$$
\begin{aligned}
\mathcal{L}_{\mathrm{VSM}}\left(\sigma, A_{\mu}\right) & =\frac{1}{2}\left(\partial_{\mu} \sigma\right)^{2}+e \varepsilon^{\mu \nu} \partial_{\mu} \sigma A_{\nu}, \\
\mathcal{L}_{\mathrm{ASM}}\left(\phi, A_{\mu}\right) & =\frac{1}{2}\left(\partial_{\mu} \phi\right)^{2}+e g^{\mu \nu} \partial_{\mu} \sigma A_{\nu}+\frac{1}{2} A_{\mu}^{2} .
\end{aligned}
$$

The gauge invariance for $a+b=2$ is now explicit.

At this point we observe that, contrary to what one might think based on the claims in $[19,23]$, it is not really necessary to perform the soldering of the two chiral bosons in order to accomplish the full local gauge invariance. Since the usual soldering procedure obtained after the addition of an interference term, produces only the vector Schwinger model, the result (9) suggests the existence of a second soldering procedure giving rise to the axial Schwinger model. Indeed, that is what we have found as we next show.

The actions $W_{+}$and $W_{-}$only depend on $\phi$ and $\rho$ through derivatives and therefore are invariant under rigid translations of these fields, the basic idea in the soldering procedure is to join the actions $W_{+}$and $W_{-}$into a new one while promoting this symmetry to a local form. Let us suppose the local variations

$$
\delta \phi=\eta(x) ; \quad \delta \rho=\alpha \eta(x),
$$

where $\alpha$ is, at this point, an arbitrary constant. In the usual soldering procedure one assumes $\alpha=1$. Under (11) we have:

$$
\delta\left(W_{+}+W_{-}\right)=\int\left(J_{+} \partial_{-} \eta+J_{-} \partial_{+} \eta\right) d^{3} x,
$$

with

$$
J_{+}=\partial_{+} \phi+e A_{+}, \quad J_{-}=\alpha\left(\partial_{-} \rho+e A_{-}\right) .
$$

Introducing two auxiliary fields $B_{ \pm}$such that

$$
\delta B_{ \pm}=-\partial_{ \pm} \eta,
$$

we have

$$
\begin{aligned}
\delta\left(\mathcal{L}_{+}\right. & \left.+\mathcal{L}_{-}+B_{+} J_{-}+B_{-} J_{+}\right)=B_{-} \partial_{+} \eta+\alpha^{2} B_{+} \partial_{-} \eta \\
& =-\delta\left(B_{+} B_{-}\right)+\left(1-\alpha^{2}\right) B_{+} \delta B_{-} .
\end{aligned}
$$

Therefore, for $\alpha= \pm 1$ we can define a soldered Lagrangian density, invariant under (11) and (14), which is given by

$$
\mathcal{L}_{\alpha}^{(s)}=\mathcal{L}_{+}+\mathcal{L}_{-}+B_{+} J_{-}+B_{-} J_{+}+B_{+} B_{-} .
$$

Eliminating the auxiliary fields through their equations of motion, we have

$$
\begin{aligned}
\mathcal{L}_{\alpha}^{(s)}= & \mathcal{L}_{+}+\mathcal{L}_{-}-J_{+} J_{-} \\
= & \partial_{+} \Phi \partial_{-} \Phi+e\left(A_{+} \partial_{-} \Phi-\alpha A_{-} \partial_{+} \Phi\right) \\
& +\frac{e^{2}(a+b-2 \alpha)}{4} A_{+} A_{-},
\end{aligned}
$$

where $\Phi=\phi-\alpha \rho$ is a field combination invariant under
(11). In both cases $\alpha=+1$ and $\alpha=-1$, if we choose $a+$ $b=2$, we recover the vector and the axial Schwinger models, respectively.

$$
\begin{aligned}
\mathcal{L}_{+1}^{(s)} & =\frac{1}{2}\left(\partial_{\mu} \Phi\right)^{2}+e \varepsilon^{\mu \nu} \partial_{\mu} \Phi A_{\nu}, \\
\mathcal{L}_{-1}^{(s)} & =\frac{1}{2}\left(\partial^{\mu} \Phi+e A^{\mu}\right)^{2} .
\end{aligned}
$$

Thus, the new soldering found here for $\alpha=-1$ generates the missing part in the rotated direct sum (9). In the two cases $\alpha= \pm 1$, the soldering procedure has produced a Lorentz covariant and local gauge invariant Lagrangian out of two anomalous gauge models which possessed only a semilocal gauge invariance. It is remarkable that in order to prove gauge invariance of $\mathcal{L}_{-1}^{(s)}$ under $\delta A_{\mu}=\partial_{\mu} \eta$ we also need to vary the scalar composite field $\delta \Phi=-e \eta$ which was invariant from the start under the transformation (11). Even in the case of $\mathcal{L}_{+1}^{(s)}$ it is assumed in the soldering approach [11] that $\delta A_{\mu}=0$ and one ends up with a local gauge invariance with $\delta A_{\mu}=\partial_{\mu} \eta \neq 0$. Thus, the connection between the original soldering symmetry and the final gauge symmetry is rather mysterious in both cases $\alpha=$ \pm 1 . We will see later that the same phenomenon appears in $d=3$ dimensions. Regarding the choice $a+b=2$ we point out that it is in agreement with the unitarity bound $a \geq 1$ and $b \geq 1$ of the chiral Schwinger models. As argued in [11], the same result $a=1=b$ could be found by using a left/right symmetry $( \pm \leftrightarrow \mp)$, implying that $a=b$ in the chiral Lagrangian densities and the condition $a+b=2$, with no need of unitarity arguments. We remark that although the models (18) are not equivalent, by functionally integrating the soldering field $\Phi$ in (17) and using $a+b=2$ we obtain an $\alpha$-independent gauge invariant effective action which, after adding the Maxwell Lagrangian density, describes a massive photon:

$$
\mathcal{L}_{\text {eff }}\left[A_{\mu}\right]=-\frac{1}{4} F^{\mu \nu} \frac{\left(\square+e^{2} / \pi\right)}{\square} F_{\mu \nu} .
$$

It is important to point out that one of the motivations to introduce the usual soldering procedure $(\alpha=1)$ is to offer a systematic way of mixing two bosonized theories, see [11], in agreement with the chiral factorization identity of fermionic determinants below:

$$
\operatorname{det}(i \not \not \partial+e \not A)=\operatorname{det}\left(i \not \partial+e \not \mathbb{A}_{+}\right) \operatorname{det}\left(i \not \not \partial+e \not \mathbb{A}_{-}\right) .
$$

Where $\mathscr{A}_{ \pm}=\not A P_{ \pm}$with $P_{ \pm}=\left(1 \pm \gamma_{5}\right) / 2$. A trivial direct sum of the two bosonized versions of the chiral determinants gives rise to a sum of the axial and vector Schwinger models and therefore does not reproduce the bosonized version of the vector Schwinger model as expected from the left-handed side of (20), while this is achieved by the usual soldering procedure. On the other hand, by tracing back the second soldering procedure $(\alpha=-1)$ it is easy to show that it is technically equivalent to the usual soldering of $W_{+}$and $W_{-}(-e)$ where $W_{-}(-e)$ corresponds to change 
the sign of the charge in $W_{-}$. Since we end up with the axial Schwinger model, we expect the existence of the factorization formula:

$$
\operatorname{det}\left(i \not \not D+e \not A \gamma_{5}\right)=\operatorname{det}\left(i \not \supset+e \not A_{+}\right) \operatorname{det}\left(\not \not \not-e \not A_{-}\right) .
$$

Indeed, by splitting the fermions in chiral components it is easy to derive the above identity. Therefore the second soldering nicely patches the two chiral Schwinger models in its bosonized form in agreement with the dual factorization formula (21) for the fermionic determinant. This type of relation between soldering and the factorization of fermionic determinants may be specially useful in $D=3$ where bosonization is much less developed.

Concluding, the generalized soldering procedure has produced in $D=2$ a self-consistent Lorentz covariant theory with local gauge invariance for $\alpha= \pm 1$ in agreement with our expectations based on the direct sum of the two chiral Schwinger models.

\section{IV. $D=\mathbf{3}$}

Now let us explore the generalized soldering in the higher dimensional case of two Maxwell-Chern-Simons models with opposite sign masses in the presence of a nonminimal interaction:

$$
\begin{aligned}
W_{+}= & \int d^{3} x\left[-\frac{1}{4} F_{\mu \nu}^{2}(A)+\frac{m_{+}}{2} \epsilon_{\alpha \beta \gamma} A^{\alpha} \partial^{\beta} A^{\gamma}\right. \\
& \left.+\gamma_{+} \epsilon_{\alpha \beta \gamma} J^{\alpha} \partial^{\beta} A^{\gamma}\right], \\
W_{-}= & \int d^{3} x\left[-\frac{1}{4} F_{\mu \nu}^{2}(B)-\frac{m_{-}}{2} \epsilon_{\alpha \beta \gamma} B^{\alpha} \partial^{\beta} B^{\gamma}\right. \\
& \left.+\gamma_{-} \epsilon_{\alpha \beta \gamma} J^{\alpha} \partial^{\beta} B^{\gamma}\right] .
\end{aligned}
$$

The above theories have been considered before [24], with $J_{\nu}=0$, in its dual form which corresponds to a self-dual and an anti-self-dual model. In [24] the models (22) and (23) have been soldered in the usual way $(\alpha=1)$ into a Maxwell-Chern-Simons-Proca theory. The general case $m_{+} \neq m_{-}$required the use of the equations of motion during the soldering procedure while in the special case $m_{+}=m_{-}$the whole method works off-shell. Such special case naturally appears in the bosonization of $\mathrm{QED}_{3}$ with two components fermions in the large mass limit. It has been first considered from the soldering point of view in [19] where it was shown that the parity noninvariant theories (22) and (23) are soldered into a parity invariant Maxwell theory with a Proca term. The soldered theory is a function of the composite field $A_{\mu}-B_{\mu}$. The nonminimal couplings $\gamma_{ \pm}$introduced here allow a generalization of $[19,24]$ into an interacting theory. In this sense $J_{\mu}$ play a role similar to the gauge field in the chiral Schwinger models of last section. We have chosen a nonminimal coupling with the current $J_{\mu}$ because it keeps the theory invariant under rigid translations of the gauge field which will play an important role in the soldering mechanism. Besides, the nonminimal coupling naturally appears when we search for the dual of $U(1)$ matter minimally coupled to the self-dual model as obtained in [25], see also $[26,27]$. In fact, in that case we have $\gamma_{ \pm}= \pm e / m_{ \pm}$where $e$ is the coupling appearing in the minimal coupling term. Now we start our generalized soldering procedure by lifting the rigid translation symmetry of (22) and (23) to the local form:

$$
\delta A_{\mu}=\eta_{\mu} ; \quad \delta B_{\mu}=\alpha \eta_{\mu}
$$

which imply

$$
\delta\left(W_{+}+W_{-}\right)=\int d^{3} x J_{\mu \nu} \partial^{\mu} \eta^{\nu}
$$

where

$$
\begin{aligned}
J_{\mu \nu}= & -F_{\mu \nu}(A)-\alpha F_{\mu \nu}(B)+\epsilon_{\mu \nu \gamma}\left(m_{+} A^{\gamma}-\alpha m_{-} B^{\gamma}\right) \\
& +\left(\alpha \gamma_{-}+\gamma_{+}\right) \epsilon_{\mu \nu \gamma} J^{\gamma}
\end{aligned}
$$

As usually one introduces auxiliary fields $B_{\mu \nu}$ such that

$$
\delta B_{\mu \nu}=-\partial_{\mu} \eta_{\nu}
$$

It is easy to derive:

$$
\begin{aligned}
\delta\left(W_{+}+\right. & \left.W_{-}+\int d^{3} x B_{\mu \nu} J^{\mu \nu}\right) \\
= & \delta\left[\frac{\left(1+\alpha^{2}\right)}{2} \int d^{3} x B_{\mu \nu}\left(B^{\mu \nu}-B^{\nu \mu}\right)\right] \\
& +\int d^{3} x\left(m_{+}-\alpha^{2} m_{-}\right) \epsilon_{\mu \nu \gamma} B^{\mu \nu} \eta^{\gamma}
\end{aligned}
$$

Since $\eta^{\gamma}$ can not be written as a local function of $\delta B_{\mu \nu}$ we choose at this point

$$
\alpha= \pm \sqrt{\frac{m_{+}}{m_{-}}}
$$

Consequently we are able to build an invariant action under the local translations (24). After the elimination of the auxiliary fields $B_{\mu \nu}$ through their equations of motion the reader can check that we arrive at:

$$
W_{\alpha}^{(S)}=W_{+}+W_{-}+\int d^{3} x \frac{J^{\mu \nu} J_{\mu \nu}}{4\left(1+\alpha^{2}\right)} \equiv \int d^{3} x \mathcal{L}_{\alpha}^{(S)}
$$

where after some rearrangements we can write down:

$$
\begin{aligned}
\mathcal{L}_{\alpha}^{(S)}= & -\frac{F_{\mu \nu}^{2}(C)}{4\left(1+\alpha^{2}\right)}+\frac{\left(m_{+}-m_{-}\right)}{2\left(1+\alpha^{2}\right)} \epsilon_{\mu \nu \gamma} C^{\mu} \partial^{\nu} C^{\gamma} \\
& +\frac{1}{2\left(1+\alpha^{2}\right)}\left[\alpha m_{-} C_{\mu}+\left(\alpha \gamma_{-}+\gamma_{+}\right) J_{\mu}\right]^{2} \\
& +\frac{\left(\alpha \gamma_{+}-\gamma_{-}\right)}{1+\alpha^{2}} \epsilon_{\mu \nu \gamma} J^{\mu} \partial^{\nu} C^{\gamma}
\end{aligned}
$$


The composite soldering field $C_{\mu}=\alpha A_{\mu}-B_{\mu}$ is invariant under the local transformations (24). Observe that no use has been made of the equations of motion contrary to [24]. In (31) we still have the two choices $\alpha=$ $\pm \sqrt{m_{+} / m_{-}}$. Suppressing the interaction $J_{\mu}=0$ we recover the same Maxwell-Chern-Simons-Proca of [24]. In parallel with the $d=2$ results of last section, the dependence on $\alpha$ only appears through the interacting terms which now contain a minimal coupling $J \cdot C$, a Thirring term $J^{2}$ and a nonminimal coupling of the Pauli type $\epsilon_{\mu \nu \gamma} J^{\mu} \partial^{\nu} C^{\gamma}$. Besides the soldering symmetry (24), if we think of $J_{\mu}$ as a dynamical field, the soldered Lagrangian (31) is symmetric under:

$$
J_{\mu} \rightarrow J_{\mu}-\partial_{\mu} \eta \quad C_{\mu} \rightarrow C_{\mu}+\frac{\alpha}{m_{+}}\left(\alpha \gamma_{-}+\gamma_{+}\right) \partial_{\mu} \eta
$$

For $\alpha \gamma_{-}+\gamma_{+} \neq 0$ the symmetry (32) is larger than the originally envisaged soldering symmetry (24) in analogy with the case $\alpha=-1$ of last section. In the $d=2$ example the two soldering choices $\alpha= \pm 1$ have led to different actions but the same effective action for the electromagnetic field $A_{\mu}$. Now in $d=3$ if we Gaussian integrate over the soldering field $C_{\mu}$ in the path integral we derive from (31) the effective Lagrangian:

$$
\begin{aligned}
\mathcal{L}_{\mathrm{eff}}^{(S)}[J]= & \frac{m_{-}\left(\gamma_{+}^{2}+\gamma_{-}^{2}\right) J^{\mu}\left(\square+m_{+} m_{-}\right) \square \theta_{\mu \nu} J^{\nu}}{\left(m_{+}+m_{-}\right)\left[\left(\square+m_{+} m_{-}\right)^{2}+\left(m_{+}-m_{-}\right)^{2} \square\right]}+\frac{m_{-}\left(m_{+}-m_{-}\right)\left(\alpha \gamma_{-}+\gamma_{+}\right)^{2} J^{2}}{2\left(m_{+}+m_{-}\right)^{2}} \\
& +\frac{m_{-} J^{\mu}\left[\left(m_{-} \gamma_{-}^{2}-m_{+} \gamma_{+}^{2}\right) \square+m_{+} m_{-}\left(m_{+} \gamma_{-}^{2}-m_{-} \gamma_{+}^{2}\right)\right] \epsilon_{\mu \nu \gamma} \partial^{\nu} J^{\gamma}}{\left(m_{+}+m_{-}\right)\left[\left(\square+m_{+} m_{-}\right)^{2}+\left(m_{+}-m_{-}\right)^{2} \square\right]}
\end{aligned}
$$

where $\theta_{\mu \nu}=g_{\mu \nu}-\partial_{\mu} \partial_{\nu} / \square$. Thus, in general the two possible choices for $\alpha$ lead us to inequivalent theories due to the second term in (33) even after integration over the soldering field. Only in the special case $m_{+}=m_{-} \equiv$ $m\left(\alpha^{2}=1\right)$ we have the same result for both choices $\alpha=$ \pm 1 , although the Lagrangians before the integration over the soldering field are different for $\alpha=1$ and $\alpha=-1$. In particular, for $\gamma_{-}=-\gamma_{+} \equiv \gamma$ we have

$$
\begin{gathered}
\mathcal{L}_{\alpha=1}^{(S)}=-\frac{F_{\mu \nu}^{2}}{8}+\frac{m^{2} C^{2}}{4}-\gamma \epsilon_{\mu \nu \gamma} J^{\mu} \partial^{\nu} C^{\gamma} \\
\mathcal{L}_{\alpha=-1}^{(S)}=-\frac{F_{\mu \nu}^{2}}{8}+\frac{m^{2} C^{2}}{4}+m \gamma J \cdot C+\gamma^{2} J^{2}
\end{gathered}
$$

We see that a nonminimal coupling of the Pauli type for $\alpha=1$ is traded in a nonminimal coupling plus a Thirring term for $\alpha=-1$. It deserves a comment the fact that such correspondence is known to appear in the dual theory of a self-dual model minimally coupled to $U(1)$ matter fields, see [25-27], which corresponds to a Maxwell-ChernSimons theory nonminimally coupled to $U(1)$ matter fields plus a Thirring interaction. The similarity with the $d=2$ case is remarkable. In that case the interaction term $e \epsilon^{\mu \nu} \partial_{\mu} \Phi A_{\nu}$ has been traded in $e \partial_{\mu} \Phi A^{\mu}+e^{2} A^{2} / 2$ as if the complementary solderings $\alpha=1$ and $\alpha=-1$ were generating dual theories. This point certainly demands a deeper investigation.

It is interesting to note that when we drop the spatial dependence of the vector fields in (22) and (23) and set $\gamma_{ \pm}=0$ we recover the Lagrangians $\mathcal{L}_{ \pm}=(1 / 2)\left[\dot{x}_{i \pm} \pm\right.$ $\left.\omega_{ \pm} \epsilon_{i j} x_{i \pm} \dot{x}_{j \pm}\right]$ where we have relabelled $A_{i} \rightarrow x_{i+} ; B_{i} \rightarrow$ $x_{i-}$ and $m_{ \pm} \rightarrow \omega_{ \pm}$. The Lagrangians $\mathcal{L}_{+}$and $\mathcal{L}_{-}$describe a right and left moving particle on a plane in the presence of a constant magnetic field orthogonal to the plane and pointing in opposite directions, respectively. Those Lagrangians have been considered before in $[19,28,29]$ and soldered in [24] where use has been made of the equations of motion for $\omega_{+} \neq \omega_{-}$. If we choose $\delta x_{i+}=$ $\eta_{i}$ and $\delta x_{i-}=\alpha \eta_{i}$ with $\alpha^{2}=\omega_{+} / \omega_{-}$we end up with a soldered Lagrangian which represents a two dimensional Harmonic oscillator in the presence of a residual magnetic field which disappears for $m_{+}=m_{-}$, i.e., in terms of the soldering field $\Phi_{i}=\left(\alpha x_{i+}-x_{i-}\right) / \sqrt{1+\alpha^{2}}$ one has $\mathcal{L}^{(S)}=(1 / 2)\left[\dot{\Phi}^{2}+\left(\omega_{+}-\omega_{-}\right) \epsilon^{i j} \Phi_{i} \dot{\Phi}_{j}-\omega_{+} \omega_{-} \Phi_{i}^{2}\right]$ in agreement with the final result of [24] but we stress that our generalized soldering procedure differently from [24] does not require the use of equations of motion, except of course for the auxiliary fields whose equations of motion are actually mathematical identities without dynamical content.

\section{CONCLUSION}

Chiral Schwinger models possess only a semilocal form of gauge invariance. However, it is known that one can recover full local gauge invariance by soldering two opposite chirality chiral Schwinger models. In this case one ends up with a vector Schwinger model. We have shown here that a direct sum of both chiral models already furnishes a local gauge invariant theory with no need of a soldering procedure. After a simple rotation of the fields the resulting theory becomes a vector plus an axial Schwinger model so suggesting the existence of a second (generalized) soldering procedure leading us to the vector Schwinger model. Indeed, we have found here a generalization of the soldering mechanism leading either to the axial or to the vector models depending on a constant 
parameter $\alpha= \pm 1$. The same twofold generalization occurs in $d=3$ when we solder two Maxwell-Chern-Simons theories with opposite sign masses $m_{+}$and $-m_{-}$. In this case we have $\alpha= \pm \sqrt{m_{+} / m_{-}}$. By allowing $\alpha \neq 1$ we have been able to implement the soldering algorithm offshell even for $m_{+} \neq m_{-}$so generalizing previous results in the literature. Introducing a nonminimal interaction of the Maxwell-Chern-Simons fields with a vector current we obtain different soldered theories for the two different choices for $\alpha$. However, in the special case $\alpha= \pm 1$ in both $d=2$ and $d=3$ we have the same theory after integration over the soldering field. Apparently, the cases $\alpha=1$ and $\alpha=-1$ generate dual versions of the same theory as we have argued in the last section. For the new soldering found here $(\alpha=-1)$ the resulting gauge symmetry is surprisingly larger than the originally imposed soldering symmetry. In order to get a deeper understanding of this and other aspects of such complementary solderings we are now applying it to the cases of nonabelian gauge theories in $d=2$, models including coupling to $2 d$ gravity and electromagnetic duality in $d=4$ which have all been considered from the point of view of usual soldering $(\alpha=$ 1). Clearly, a better understanding of the generalized soldering from the path integral point of view through a possible master action approach would probably unravel some of the interesting features of this mechanism.

\section{ACKNOWLEDGMENTS}

This work is partially supported by Conselho Nacional de Pesquisa e Desenvolvimento (CNPq).
[1] D. J. Gross, J. A. Harvey, E. Martinec, and R. Rohm, Phys. Rev. Lett. 54, 502 (1985); Nucl. Phys. B256, 253 (1985); B267, 75 (1986).

[2] M. Gomes, V. Kurak, V. O. Rivelles, and A. J. da Silva, Phys. Rev. D 38, 1344 (1988).

[3] W. Siegel, Nucl. Phys. B238, 307 (1984).

[4] R. Floreanini and R. Jackiw, Phys. Rev. Lett. 59, 1873 (1987).

[5] X. G. Wen, Phys. Rev. Lett. 64, 2206 (1990); Phys. Rev. B 41, 012838 (1990); M. Stone, Phys. Rev. B 42, 212 (1990).

[6] H. O. Girotti, M. Gomes, V. Kurak, V. O. Rivelles, and A. J. da Silva, Phys. Rev. Lett. 60, 1913 (1988).

[7] M. Stone, Illinois report No. ILL-(TH)-89-23, 1989 (unpublished); Phys. Rev. Lett. 63, 731 (1989); Nucl. Phys. B327, 399 (1989); D. Depireux, S. J. Gates, Jr., and Q-Han Park, Phys. Lett. B 224, 364 (1989); E. Witten, Commun. Math. Phys. 144, 189 (1992).

[8] A. A. Kirilov, Elements of The Representation Theory (Nakua, Moscow, 1972); A. Alekseev, L. Faddeev, and S. Shatashvili, J. Geom. Phys. 5, 391 (1988); A. Alekseev and S. Shatashvili, Nucl. Phys. B323, 719 (1989).

[9] E. Witten, Commun. Math. Phys. 92, 455 (1984).

[10] R. Amorim, A. Das, and C. Wotzasek, Phys. Rev. D 53, 5810 (1996).

[11] E. M. C. Abreu, R. Banerjee, and C. Wotzasek, Nucl. Phys. B509, 519 (1998).

[12] J. Schwinger, Phys. Rev. 128, 2425 (1962).

[13] R. Jackiw and R. Rajaraman, Phys. Rev. Lett. 54, 1219
(1985).

[14] E. M. C. Abreu and C. Wotzasek, Phys. Rev. D 58, 101701 (1998).

[15] S. J. Gates, Jr. and W. Siegel, Phys. Lett. B 206, 631 (1988).

[16] C. M. Hull, Phys. Lett. B 206, 234 (1988); Phys. Lett. B 212, 437 (1988).

[17] P. K. Townsend, K. Pilch, and P. van Nieuwenhuizen, Phys. Lett. B 136, 38 (1984).

[18] S. Deser and R. Jackiw, Phys. Lett. B 139, 371 (1984).

[19] R. Banerjee and C. Wotzasek, Nucl. Phys. B527, 402 (1998).

[20] J. Schwarz and A. Sen, Nucl. Phys. B411, 35 (1994).

[21] A. de Souza Dutra, Phys. Lett. B 286, 285 (1992).

[22] A. Pressley and G. Segal, Loup Groups (Oxford University Press, Oxford, 1986).

[23] For a review see: C. Wotzasek, hep-th 9806005.

[24] R. Banerjee and S. Kumar, Phys. Rev. D 60, 085005 (1999).

[25] M. Gomes, L. C. Malacarne, and A. J. da Silva, Phys. Lett. B 439, 137 (1998).

[26] M. A. Anacleto, A. Ilha, J. R. S. Nascimento, R. F. Ribeiro, and C. Wotzasek, Phys. Lett. B 504, 268 (2001).

[27] D. Dalmazi, J. Phys. A 37, 2487 (2004).

[28] G. V. Dunne, R. Jackiw, and C. A. Trugenberger, Phys. Rev. D 41, 661 (1990).

[29] R. Banerjee and S. Ghosh, J. Phys. A 31, L603 (1998). 